\title{
QUANTIFYING THE BEHAVIOUR OF CURVATURE PERTURBATIONS NEAR HORIZON CROSSING
}

\author{
ELLIE NALSON*, IAN HUSTON and KARIM A. MALIK \\ Astronomy Unit, Queen Mary University of London, Mile End Road, London, UK \\ * Email: e.nalson@qmul.ac.uk \\ ADAM J. CHRISTOPHERSON \\ School of Physics and Astronomy, University of Nottingham, University Park, Nottingham, \\ $N G 7$ 2RD, UK
}

\begin{abstract}
How much does the curvature perturbation change after it leaves the horizon, and when should one evaluate the power spectrum? To answer these questions we study single field inflation models numerically, and compare the evolution of different curvature perturbations from horizon crossing to the end of inflation. We find that e.g. in chaotic inflation, the amplitude of the comoving and the curvature perturbation on uniform density hypersurfaces differ by up to $180 \%$ at horizon crossing assuming the same amplitude at the end of inflation, and that it takes approximately 3 efolds for the curvature perturbation to be within $1 \%$ of its value at the end of inflation.
\end{abstract}

Keywords: Inflation, Cosmological Perturbations

\section{Introduction}

It is well known that the curvature perturbations on both uniform density hypersurfaces, $\zeta$, and on comoving hypersurfaces, $\mathcal{R}$, are conserved on large scales. The standard approach used to calculate the power spectrum of perturbations after horizon crossing assumes that the limit $k \rightarrow 0$ has been reached, see Ref ${ }^{1}$ However, immediately after horizon crossing the wavenumber will not yet have become sufficiently small for this limit to be accurate and gradient terms will still play a role. Exactly how long this evolution will last and the size of the errors if the curvature perturbation is evaluated too early are issues addressed in this work ${ }^{2}$

\section{Equations and Numerics}

We consider single field inflation in a homogeneous, isotropic, Friedmann-RobertsonWalker background, with scalar perturbations of the field and metric to first order, and work in flat gauge and Fourier space. The resulting first order Klein-Gordon equations are solved numerically by following Salopek et al ${ }^{3}$ Initial conditions for the background are selected for each potential following Huston 4 and at early times we assume the Bunch-Davies vacuum ${ }^{3 / 4}$ We map the initial scalar field fluctuations, onto conserved quantities, that remain constant in the limit $k \rightarrow 0$ for adiabatic perturbations. We focus on the curvature perturbation on uniform density hypersurfaces $\zeta \equiv \psi+\frac{\mathcal{H}}{\rho_{0}^{\prime}} \delta \rho$, and the comoving curvature perturbation $\mathcal{R} \equiv \psi+\frac{\mathcal{H}}{\varphi_{0}^{\prime}} \delta \varphi$ (see e.g. Ref. (5). These two gauge-invariant curvature perturbations, are related by a constraint equation and $\zeta+\mathcal{R}$ becomes small on super-horizon scales. We show plots for the modes $k_{1}=2.77 \times 10^{-5} M p c^{-1}, k_{2}=2.00 \times 10^{-3} M p c^{-1}$ (WMAP pivot scale) 
and $k_{3}=1.45 \times 10^{-1} \mathrm{Mpc}^{-1}$. The results below are for the potential $U=\frac{1}{2} m^{2} \varphi^{2}$ with $m=6.32 \times 10^{-6} M_{\mathrm{PL}}$ but we also obtain results for $U=U_{0}+\frac{1}{2} m^{2} \varphi^{2}, U=\frac{1}{4} \lambda \varphi^{4}$ and $U=\sigma \varphi^{2 / 3}$. For full details see Nalson et al. ${ }^{2}$ Our numerical results have been verified with a second numerical program, pyflation!

To deduce an analytic expression for the curvature perturbation, we follow Ref. 7 The Eq. (3.34) given there is only valid in the large scale limit, however, it must be evaluated exactly when the corresponding mode crosses the horizon.

\section{Results}

As expected, we can see from Fig. 1(a) that a short time after horizon crossing there is no longer any appreciable evolution in either the power spectrum of $\zeta, \mathcal{P}_{\zeta}(k)$ or the power spectrum of $\mathcal{R}, \mathcal{P}_{\mathcal{R}}(k)$, but that there is some evolution immediately after horizon crossing. We find that despite $\zeta$ and $\mathcal{R}$ being equivalent very far outside the horizon, the difference between $|\mathcal{R}|$ and $|\zeta|$ at horizon crossing can be as much as $20 \%$ and $\zeta$ remains significantly larger for at least a couple of efolds. We also find that the error in evaluating the power spectra numerically at horizon crossing rather than using the correct analytic expression or the full numerical solution at late times can be as much as $180 \%$ for $\mathcal{P}_{\zeta}(k)$ and $100 \%$ for $\mathcal{P}_{\mathcal{R}}$ (this is expected analytically $\left.{ }^{8}\right)$. Lastly we showed that to evaluate the power spectra without using the analytic expression, one would need to wait 3.2 and 2.9 efolds to ensure the answer for $\mathcal{P}_{\mathcal{R}}(k)$ and $P_{\zeta}$, respectively, are correct to within $1 \%$ of the value at the end of inflation. Similar results were obtained for the other potentials.

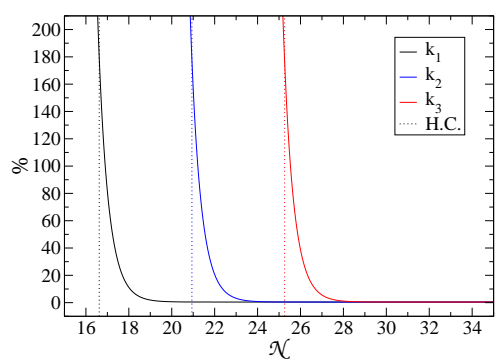

(a) Percentage difference between $\mathcal{P}(k)$ evaluated, numerically, at the end of inflation and at each time step, plotted for $k_{1}$ (black, left), $k_{2}$ (blue, middle) and $k_{3}$ (red, right).

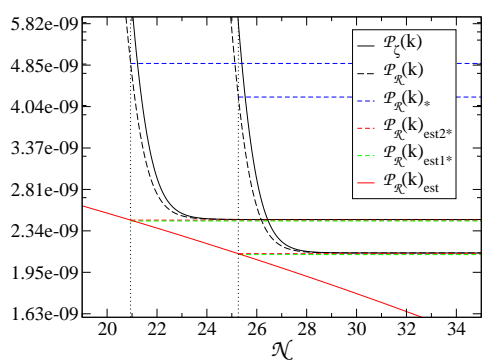

(b) Numerical solutions compared to correct analytic solution and naïve evaluation of the power spectra at horizon crossing, plotted for $k_{2}$ (left lines) and $k_{3}$ (right lines).

Fig. 1. The evolution of $\mathcal{P}_{\mathcal{R}}(k)$ and $\mathcal{P}_{\zeta}(k)$ are plotted against the number of efolds, $\mathcal{N}$.

In Fig. 1 we compare the correct analytic solution and the naïve calculation of the power spectrum at horizon crossing with the numerical solutions. When we compare the numerical solution to $\mathcal{P}_{\mathcal{R}}(k)$ evaluated at horizon crossing we find that there is a $100 \%$ error in our answer. We also see that if one were to use the analytic expression, but to evaluate it 'some efolds after horizon crossing' rather than at 
horizon crossing one would underestimate the amplitude of the power spectrum. This corresponds to following the red line in Fig. 1(b) e.g. evaluating the analytic expression 4 efolds after horizon crossing incurs a $10 \%$ error. Lastly we see that the analytic and numerical expressions do not agree with each other shortly after horizon crossing, one must wait at least 3.2 efolds for these two values to agree.

\section{Conclusions}

We have quantified the evolution of the curvature perturbations after inflation and highlighted possible errors which can occur. As we are entering an era where we hope to constrain parameters to within a percent using e.g. Planck data, it is of particular importance that these errors are both minimised and quantified. We show the difference between analytic and numerical expressions close to the horizon. The numerical results, instantaneous values of the power spectrum at horizon crossing, while not of observational significance, are useful in many ways, e.g. as initial conditions for other analytical or numerical schemes operating outside the horizon. If we are interested in late time values we should not evaluate numerical results at horizon crossing, as unlike the analytic results they will not be accurate. If we are interested in the instantaneous values at or close to horizon crossing, the analytic expressions are no longer valid and one must use numerical methods. For example, this is important if there is a second phase of evolution which starts to dominate during the first three efolds after horizon crossing. In conclusion, we have highlighted that confusion between the different curvature perturbations, how they are evaluated and when each expression is valid can introduce additional errors when comparing theoretical results with observations.

\section{Acknowledgements}

EN is funded by a STFC studentship. AJC is funded by the Sir Norman Lockyer Fellowship of the Royal Astronomical Society, IH is funded by the STFC under Grant ST/G002150/1, and KAM is supported in part by the STFC under Grants ST/G002150/1 and ST/H002855/1.

\section{References}

1. E. J. Copeland, E. W. Kolb, A. R. Liddle and J. E. Lidsey, Phys.Rev. D48, 2529 (1993).

2. E. Nalson, A. J. Christopherson, I. Huston and K. A. Malik, Class.Quant.Grav, (in press).

3. D. Salopek, J. Bond and J. M. Bardeen, Phys.Rev. D40, p. 1753 (1989).

4. I. Huston, Constraining Inflationary Scenarios with Braneworld Models and Second Order Cosmological Perturbations (PhD Thesis) (2010).

5. K. A. Malik and D. Wands, Phys.Rept. 475, 1 (2009).

6. I. Huston and K. A. Malik, JCAP 1110, p. 029 (2011).

7. J. E. Lidsey, A. R. Liddle, E. W. Kolb, E. J. Copeland, T. Barreiro et al., Rev.Mod.Phys. 69, 373 (1997).

8. D. Polarski and A. A. Starobinsky, Class.Quant.Grav. 13, 377 (1996). 